Pulse-train solutions and excitability in an optoelectronic oscillator

This article has been downloaded from IOPscience. Please scroll down to see the full text article.

2011 EPL 9634001

(http://iopscience.iop.org/0295-5075/96/3/34001)

View the table of contents for this issue, or go to the journal homepage for more

Download details:

IP Address: 152.3.183.177

The article was downloaded on 21/11/2011 at 22:14

Please note that terms and conditions apply. 


\title{
Pulse-train solutions and excitability in an optoelectronic oscillator
}

\author{
D. P. $\operatorname{Rosin}^{1,2(a)}$, K. E. Callan ${ }^{1}$, D. J. Gauthier ${ }^{1}$ and E. Schöll ${ }^{2}$ \\ ${ }^{1}$ Department of Physics, Duke University - Durham, NC 27708, USA \\ ${ }^{2}$ Institut für Theoretische Physik, Technische Universität Berlin - Hardenbergstr. 36, D-10623 Berlin, Germany, EU
}

received 19 June 2011; accepted in final form 7 September 2011

published online 12 October 2011

PACS 42.82.Fv - Hybrid systems

PACS 05.45.-a - Nonlinear dynamics and chaos

PACS 87.19.lr - Control theory and feedback

\begin{abstract}
We study an optoelectronic time-delay oscillator with bandpass filtering for different values of the filter bandwidth. Our experiments show novel pulse-train solutions with pulse widths that can be controlled over a three-order-of-magnitude range, with a minimum pulse width of $\sim 150 \mathrm{ps}$. The equations governing the dynamics of our optoelectronic oscillator are similar to the FitzHugh-Nagumo model from neurodynamics with delayed feedback in the excitable and oscillatory regimes. Using a nullclines analysis, we derive an analytical proportionality between pulse width and the low-frequency cutoff of the bandpass filter, which is in agreement with experiments and numerical simulations. Furthermore, the nullclines help to describe the shape of the waveforms.
\end{abstract}

Copyright (c) EPLA, 2011

Introduction. - Excitability is an essential characteristic of many biological systems, such as neural networks and the heart [1]. The FitzHugh-Nagumo (FHN) model [2] is a canonical model of excitability, which exhibits a variety of dynamics ranging from spiking to relaxation oscillations. The study of excitability in optics and electronics is of great current interest [3-6].

There is also great interest in developing devices that produce periodic trains of optical pulses, which correspond to distinct comb lines in the frequency domain, for applications ranging from metrology [7] to frequency conversion and signal broadcasting [8]. Narrow pulses, with correspondingly large bandwidths, are particularly useful. Therefore, the ability to tune pulse widths to short time scales is very desirable.

In this letter, we describe novel pulse-train solutions generated by a time-delay optoelectronic oscillator (OEO). Using nullclines corresponding to solutions that are periodic with the time delay, we find an analytic expression relating the pulse width to the low-frequency cutoff of the bandpass filter in our system. The analytic expression is in good agreement with experiments and numerical simulations. We also apply a similar analysis to the oscillatory regime, where we can control the duty cycle of the limit-cycle oscillations. Again, the nullclines make analytical studies of the waveforms of these solutions possible.

(a) E-mail: rosin@phy .duke.edu
Furthermore, we find that the periodic nullclines of our model are similar to those of the FHN model, which exhibits features of excitability, such as a thresholdtriggered response, for certain parameter values. Thus, our system shows similar trajectories in phase space that correspond to a time series of a fast rise and fall of the signal (a pulse), followed by a slower refractory period. Due to the time-delayed feedback, our system behaves similar to the FHN model with delayed feedback [9-11], where pulse trains can be excited if the system is initially perturbed above a threshold.

The nullcline analysis provides a simple method for characterizing low-feedback dynamic states in the OEO. We also see many applications for these pulsing states, such as a microwave frequency comb generator, an optoelectronic memory device, or a building block for an artificial neural network.

Experimental setup and model. - In our optoelectronic oscillator, schematically shown in fig. 1, the beam generated by a $1.55 \mu \mathrm{m}$ wavelength semiconductor laser is coupled into a single-mode optical fiber, passes through a polarization controller, and is injected to a Mach-Zehnder modulator (MZM). The optical power transmission of the MZM is a nonlinear function of the applied voltage $\left(P_{\text {out }} / P_{\text {in }}\right) \propto \cos ^{2}\left(m+\pi V_{\text {rf }} / 2 V_{\pi, \text { rf }}\right)$, where $P_{\text {in }}\left(P_{\text {out }}\right)$ is the power input (output) to the modulator. We independently apply a time-dependent voltage $V_{\text {rf }}$ to the radio frequency 


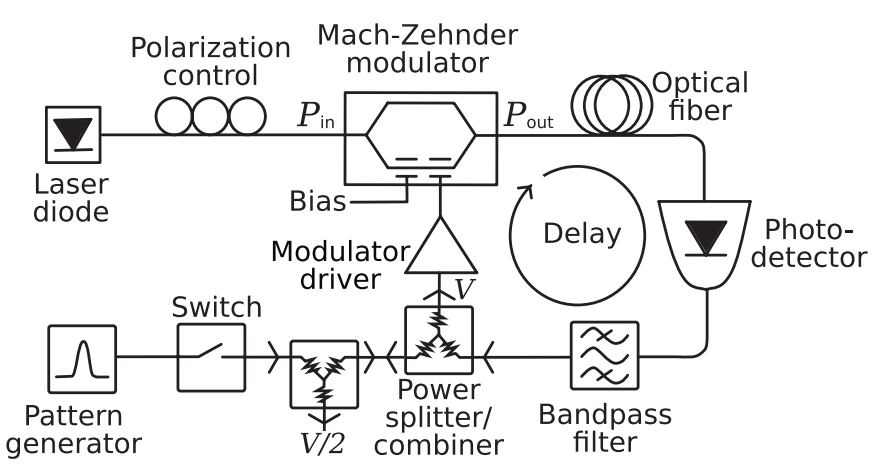

Fig. 1: Experimental setup of the optoelectronic oscillator.

(rf) port of the device (half-wave voltage $V_{\pi, \mathrm{rf}}=7.2 \mathrm{~V}$ ) and a bias to set the phase shift $m$. Light exiting the modulator passes through a delay line and is incident on an inverting photodetector. The electronic signal from the photodetector passes through a bandpass filter (which may or may not be inverting), and half of the resulting signal, denoted by $V$, is amplified by an inverting modulator driver (MD, gain $g_{\mathrm{MD}} \approx-17$ ) and fed back to the MZM via the rf input port. The MD saturates at a voltage of $V_{\text {sat }} \approx 4.8 \mathrm{~V}$, and is modeled by a hyperbolic tangent function. Thus, the overall feedback signal at the input of the MD is given by

$$
F(V)=\gamma g / d\left\{\cos ^{2}[m+d \tanh (V / g)]-\cos ^{2}[m]\right\},
$$

where $d=\pi V_{\text {sat }} / 2 V_{\pi, \mathrm{rf}}=1.0$ and $g=V_{\text {sat }} / g_{\mathrm{MD}}=-0.28 \mathrm{~V}$ are constants and $\gamma$ is the overall feedback gain. In the appendix, we describe how we measure these parameters and adjust their values to obtain the best agreement with our model.

In the closed-loop setup, the other half of the signal leaving the bandpass filter is split again so that we can measure the dynamical variable $V / 2$ with a high-speed oscilloscope ( $8 \mathrm{GHz}$ analog bandwidth, $40 \mathrm{GSa} / \mathrm{s}$ sampling rate). To perturb the dynamics, a controllable electrical pulse from a pattern generator can be combined with $V$ at the input of the MD. The pattern generator is isolated from the system with an electrical switch.

All of the system components have bandpass characteristics; we further restrict the bandwidth using a variety of bandpass filters placed after the photodetector (table 1). It is well known that the system dynamics is affected by bandpass filtered feedback [12-16], which we model with a two-pole bandpass filter with low-(high-)frequency cutoff $\omega_{-}\left(\omega_{+}\right)$, center frequency $\omega_{0}=\sqrt{\omega_{-} \omega_{+}}$, and bandwidth $\Delta=\omega_{+}-\omega_{-} \approx \omega_{+}$. The dynamics with delayed bandpass feedback is thus described by the coupled delay differential equations (DDE),

$$
\begin{aligned}
& \dot{V}(t)=\Delta\{-V(t)-U(t)+F[V(t-\tau)]\}, \\
& \dot{U}(t)=\Delta \varepsilon V(t) .
\end{aligned}
$$

Here, the overdot denotes the derivative with respect to time, $\varepsilon=\omega_{0}^{2} / \Delta^{2}$ is a small parameter characterizing the
Table 1: Characteristics of bandpass filters used in experiment and simulation.

\begin{tabular}{c|c|c|c}
\hline Filter & $\omega_{-} /(2 \pi)(\mathrm{Hz})$ & $\omega_{+} /(2 \pi)(\mathrm{Hz})$ & $\varepsilon$ \\
\hline$\# 1$ & $52.9 \times 10^{3}$ & $1.74 \times 10^{9}$ & $3.04 \times 10^{-5}$ \\
$\# 2$ & $460 \times 10^{3}$ & $3.90 \times 10^{9}$ & $1.18 \times 10^{-4}$ \\
$\# 3$ & $1.48 \times 10^{6}$ & $463 \times 10^{6}$ & $3.22 \times 10^{-3}$ \\
$\# 4$ & $3.32 \times 10^{6}$ & $1.22 \times 10^{9}$ & $2.74 \times 10^{-3}$ \\
$\# 5$ & $28.2 \times 10^{6}$ & $1.11 \times 10^{9}$ & $2.67 \times 10^{-2}$ \\
$\# 6$ & $119 \times 10^{6}$ & $1.53 \times 10^{9}$ & $9.15 \times 10^{-2}$ \\
\hline
\end{tabular}

bandpass filter, and $F$ is the nonlinear function given in eq. (1). Previous investigations have shown that such a model is in good agreement with experiments [16-18].

In our experiments, $|\gamma|$ ranges from 0 to 10 by adjusting the laser power with an attenuator, and $|m| \leqslant \pi / 2$. We adjust the cutoff frequencies, $\omega_{-}, \omega_{+}$, by using different bandpass filters and we use three different time delays: $\tau=93 \mu \mathrm{s}, 1.5 \mu \mathrm{s}$, and $65 \mathrm{~ns}$.

Pulse-train solutions. - We observe novel pulsing solutions if the OEO is perturbed by an abovethreshold [16] electrical pulse with a width similar to the eventual pulsing solution in the vicinity of $m \approx 0$ or $m \approx \pm \pi / 2$ and $2 \lesssim|\gamma| \lesssim 3$ (depending on the bandpass characteristics) for bandpass filters listed in table 1 and time delays $\tau>2 \pi / \omega_{-}$. We emphasize that the phase shift $m$ needs to be set near maximum or minimum transmission point of the MZM and the delay needs to be longer than the refractory phase. The delayed feedback every $\tau$ causes a train of pulses to be produced with a period $\tau+\delta$, as shown in fig. 2(a), where the turn-on delay $\delta$ [9] is small compared to $\tau$ so that it can be neglected in the expression for the period. However, $\delta$ plays an important role in triggering the excitation of a pulse away from the fixed point. Pulsating dynamics can also be observed without injecting a pulse by starting in the chaotic state and then decreasing $|\gamma|$. The authors of ref. [17] observed multipulse dynamics via this method; we also see multipulse behavior coexisting with the single-pulse behavior we report on here.

The electronic pulses have a characteristic shape, as shown in fig. 2(b): the voltage increases rapidly from zero (point A) to the maximum amplitude (point B), then it decreases slowly to point $\mathrm{C}$, before it rapidly drops past zero to point $\mathrm{D}$. After the pulse, the voltage slowly recovers back to zero, the resting state of the system. Thus, the segment from A to $\mathrm{B}$ corresponds to the rise, $\mathrm{B}$ to $\mathrm{C}$ to the high level, and $\mathrm{C}$ to $\mathrm{D}$ to the fall of the pulse. The segment from D back to the fixed point is the refractory phase.

The variable $U$ cannot be directly measured in the experiment, but we can calculate $U$ with eq. (2b) from the experimentally measured values of $V$ using

$$
U(t)-U(0)=\Delta \varepsilon \int_{0}^{t} V(s) \mathrm{d} s .
$$



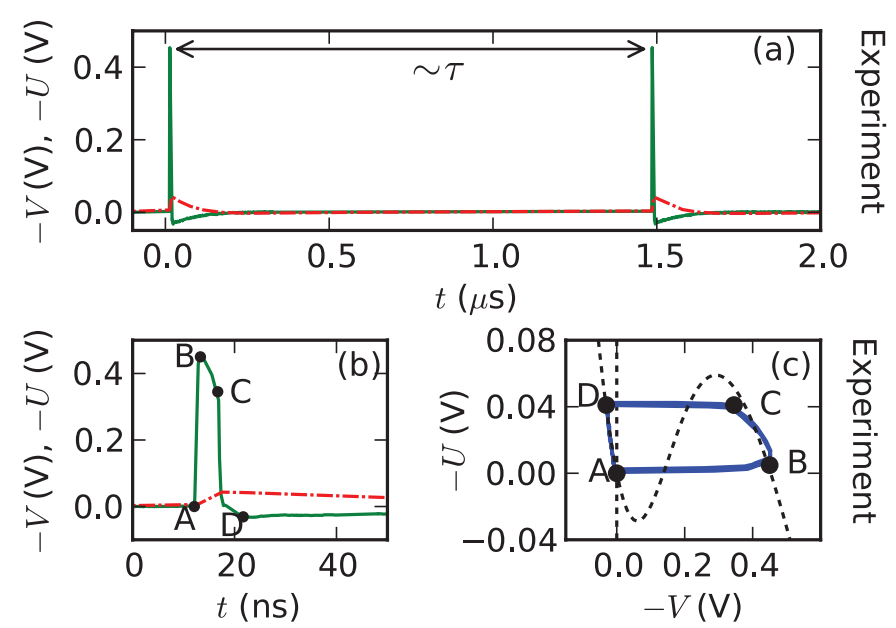


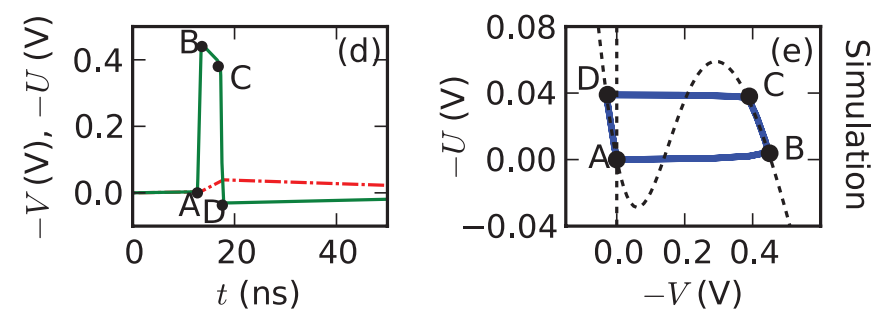

Fig. 2: (Color online) Dynamics in the pulsing regime for $m=0, \gamma=-2.54, \tau=1.5 \mu \mathrm{s}, d=1.0, g=-0.28 \mathrm{~V}$, and filter \#4. (a), (b) Experimental time series of $V(t)$ (green line) and calculated $U(t)$ (red dashed line), and (c) the associated experimental phase portrait $(V(t), U(t))$ (blue line: trajectory; black dashed lines: nullclines). (d) Numerical time series of $V(t)$ (green line) and $U(t)$ (red dashed line) and (e) associated phase portrait $(V(t), U(t))$ (blue line: trajectory; black dashed lines: nullclines). The dots A-D mark corresponding points on (b) and (c), as well as on (d) and (e), $U(\mathrm{~B})=0, U(\mathrm{C})=-0.04$. The experimental time series are averaged over 20 waveforms.

The integral is calculated with Simpson's rule with an offset $U(0)$ chosen such that $U$ approaches the fixed point right before a pulse ${ }^{1}$. The resulting waveform $U(t)$ is also shown in fig. 2(a), (b). As with $V, U$ is in the resting state at zero until point A, when the pulse in $V$ begins. Since $\varepsilon$ is small, $U$ rises much more slowly than $V$. When $V$ begins to fall, $U$ returns slowly to zero.

These pulsing solutions can be visualized in phase space by considering the nullclines corresponding to $\tau$-periodic solutions of eqs. (2). The $\tau$-periodic nullclines are found by setting $\dot{V}=0, \dot{U}=0$ and $V(t)=V(t-\tau)$, giving

$$
\begin{aligned}
U(V) & =-V+F(V), \\
V & =0 .
\end{aligned}
$$

The intersection of the nullclines, $\left(U^{*}, V^{*}\right)=(0,0)$, is the fixed point of the system for all parameter values. We note that the nullclines given by eqs. (4) were used previously [16] to predict the size of a perturbation needed to destabilize the fixed point.

\footnotetext{
${ }^{1}$ Furthermore, we subtract a small dc voltage from $V(\approx 1.3 \mathrm{mV})$, which is not amplified by the ac-coupled MD and is an offset of either the oscilloscope or photodetector.
}

Figure 2(c) shows the phase portrait $(V, U)$ corresponding to the experimental time series shown in fig. 2(b), with the nullclines (eqs. (4)) superimposed. The four points A-D from fig. 2(b) are also indicated on the trajectories in the phase plane. A pulse at time $t-\tau$ excites the trajectory starting at the fixed point (point A) to the right branch of the nonlinear nullcline (point B). The trajectory travels along the nonlinear nullcline toward the maximum (point C) and moves horizontally to the left branch of the nonlinear nullcline (point D). Finally, the trajectory travels along the left branch of the nonlinear nullcline back toward the fixed point. The motion of the trajectory along the branches of the nonlinear nullcline is due to the fact that the velocity field points upward (downward) along the right (left) branch of the nonlinear nullcline. Between the middle branch and the right (left) branch, the velocity field is directed right (left).

The small filter parameter $0<\varepsilon \ll 1$ acts as a time scale separation between changes in $V$ and $U$. Thus, the transitions from $\mathrm{A}$ to $\mathrm{B}$ and from $\mathrm{C}$ to $\mathrm{D}$ happen faster than the transitions from $\mathrm{B}$ to $\mathrm{C}$ and from $\mathrm{D}$ to $\mathrm{A}$, because, in the former, mostly $V$ (fast) is changing and, in the latter, mostly $U$ (slow) is changing.

Figures 2(d), (e) show the pulse-train solutions generated by simulation, where we numerically integrate the model equations (eq. (2)) to produce a time series (panel (d)) and associated phase portrait (panel (e) $)^{2}$. Pulse trains in the simulation and the experiment have similar waveforms, as shown in figs. 2(b), (d), and similar phase space trajectories, as shown in figs. 2(c), (e). However, the trajectory from $\mathrm{B}$ to $\mathrm{C}$ deviates from the nullcline in the experimental phase portrait due to non-ideal bandpass characteristics.

Because the trajectory of the pulse follows the nonlinear nullcline, which is a simple function, it is possible to calculate the characteristics of the pulses analytically. From the phase portraits shown in figs. 2(c), (e), we see that the nonlinear nullcline is similar to a cubic function. A cubic nullcline appears in the FHN system, which is a generic model for excitability [2] and is also capable of producing pulse-train solutions when a time-delayed feedback control is added [10]. The $\tau$-periodic nullclines help to explain the pulsing mechanism, analogous to the work by Schöll et al. for the FHN system [10].

For different parameters $\gamma$ and $m$, the nullclines change shape. Specifically, decreasing $|\gamma|$ puts the extrema of the nonlinear nullcline closer together (see eq. (4a)), leading to smaller pulse widths. When the maximum of the nonlinear nullcline is lower than $U=0$ (the fixed point), pulses cannot occur (i.e., $|\gamma| \approx 2$ for $m=0$ ). As $|\gamma|$ increases, the pulse widths become larger. If $|\gamma|$ is sufficiently large (i.e., $|\gamma| \approx 5$ for $m=0$ ) the initial pulse results in chaotic dynamics, as discussed in [16,20]. Therefore, there also exists an upper boundary in parameter space for pulsetrain solutions.

\footnotetext{
${ }^{2}$ As an integrator we used the code RADAR5 [19].
} 
Pulse-train solutions can be obtained at both $m \approx 0$ and $m \approx \pm \pi / 2$, but have opposite polarity from one another. At $m \approx \pm \pi / 2$, the nonlinear nullcline has its extrema to the left of the fixed point rather than to the right. Physically, the optical pulses with $m \approx \pm \pi / 2$ are light pulses and those with $m \approx 0$ are dark pulses, which can be explained by considering the transmission of the MZM. For $m= \pm \pi / 2$, the MZM is shut when no voltage is applied to its rf port. When an electrical pulse arrives at this port, it opens the optical transmission of the MZM for a time equal to the width of the incoming pulse, creating an optical pulse. This optical pulse is converted to an electrical pulse, which triggers another optical pulse in the MZM after the delay time $\tau$. For $m=0$ the MZM is open most of the time, but shuts when a pulse is applied, leading to dark pulses in the optical domain. The polarity of the electronic pulses depends on whether or not the bandpass filter is also inverting. For simplicity, we plot the pulses so that they appear with positive polarity.

Controlling the pulse width. - In this section, we use the nullcline description to find an analytic relation between the width $w$ of the pulses and $\omega_{-}$. A similar approach for the FHN system was undertaken by Schöll et al. [10]. Here, we estimate $w$ as the time it takes the trajectory to move from point $\mathrm{B}$ to $\mathrm{C}$ in fig. 2,

$$
w=\int_{\mathrm{B}}^{\mathrm{C}} \mathrm{d} t=\int_{U(\mathrm{~B})}^{U(\mathrm{C})} \frac{1}{\dot{U}} \mathrm{~d} U .
$$

Substituting $\dot{U}$ from eq. (2b) into eq. (5) gives

$$
w=\frac{1}{\Delta \varepsilon} \int_{U(\mathrm{~B})}^{U(\mathrm{C})} \frac{1}{V(U)} \mathrm{d} U \propto \frac{1}{\Delta \varepsilon} \approx \frac{1}{\omega_{-}},
$$

where the approximation $\Delta \varepsilon \approx \omega_{-}$holds for bandpass filters where $\omega_{+} \gg \omega_{-}$, which is in good agreement $( \pm 8 \%)$ for the filters listed in table 1 . We use the observation from simulations that the integral bounds in eq. (6) are nearly constant for filters \#1-5. With increasing $\omega_{-}$and decreasing $\omega_{+}$, we find that the fast transitions from $\mathrm{A}$ to $\mathrm{B}$ and from $\mathrm{C}$ to $\mathrm{D}$ in fig. 2 become slower and the values of $U(\mathrm{~B})$ and $U(\mathrm{C})$ move slightly up and down, respectively. The derivation shown here is universal for $\omega_{+} \gg \omega_{-}$. Using eq. (6), we can control $w$ by changing $\omega_{-}$.

We test our prediction by measuring the full width at half-maximum of pulses produced both in the experiment and simulation. Figure 3 shows that $w$ decreases as $\omega_{-}$is increased. In both experiment and simulation, $w$ follows this trend over three orders-of-magnitude.

Going beyond the proportionality given in eq. (6), we evaluate the integral, using the approximation $\tanh (V / g) \approx 1$ for $|V| \gg|g|$, which involves an error $\lesssim 5 \%$. Then the right branch of the nonlinear nullcline (eq. (4a)) can be inverted and is described by a linear function,

$$
V(U)=\frac{\gamma g}{d}\left(\cos ^{2}(m+d)-\cos ^{2}(m)\right)-U
$$



Fig. 3: Observed pulse widths of the pulse-train solutions for different bandpass filters shown in table 1 (labeled) as a function of $\omega_{-}$in the experiment (a) and simulation (b) with the analytic equation (8) superimposed as a solid line. The parameters in experiment and simulation are $m=0, d=1.0$, $g=-0.28 \mathrm{~V}, \gamma \approx \pm 2.6$, except for filter $\# 6(\gamma=2.9)$, and $\tau=$ $1.5 \mu \mathrm{s}$, except for filter \#1 and \#2 $(\tau=93 \mu \mathrm{s})$.

The lower boundary of the integral (eq. (6)) is approximately given by the fixed point $U(\mathrm{~B})=0$ and the upper boundary $U(\mathrm{C})= \pm 0.05$ is determined numerically from the simulation for the value $|\gamma|=2.6$ used in fig. 3 for filters \#1-5, leading to an analytical pulse width of

$$
w \approx-\frac{1}{\Delta \varepsilon} \ln \left(1+\frac{U(\mathrm{C}) d}{\gamma g \sin ^{2} d}\right) \approx \frac{0.1}{\Delta \varepsilon},
$$

for $m=0$.

Equation (8) is shown as solid line of slope of -1 in the double logarithmic plot of fig. 3. The results are in good agreement and match well with the analytical approximation. The small differences are due to the non-ideal bandpass characteristics in the experiments, as discussed in the appendix, which leads to an uncertainty with the determination of $\gamma$ from eq. (A.1). The deviation of the simulated data point \#6 in fig. 3(b) from the analytic equation (8) can be explained by the different value of $\gamma(=2.9)$, which leads to a change in $U(\mathrm{C})$. When $|\gamma|$ is increased, the maximum of the nonlinear nullcline (eq. (4a)), and likewise the path length from B to C in fig. 2, increases with $|\gamma|$, which changes the value of the integral in eq. (8). This leads to longer pulse widths, allowing one to tune the pulse width by a factor of $\approx 3$ for a fixed $\omega_{-}$.

The smallest pulse width recorded in the experiment $(\sim 150 \mathrm{ps})$ is artificially high due to experimental limitations. The widths were measured using an oscilloscope with an analog bandwidth of $8 \mathrm{GHz}$, corresponding to a risetime of $\approx 50 \mathrm{ps}$, making resolution of pulse widths smaller than $\sim 200$ ps impractical.

Thus, it is possible to control the pulse width over a large range from below $100 \mathrm{ps}$ to hundreds of nanoseconds by controlling the lower cutoff frequency $\omega_{-}$. Based on our simulation, even shorter pulse widths are possible for larger values of $\omega_{-}$and appropriate bandwidths $\Delta$.

Controlling the duty cycle. - As $m$ is tuned past $m_{\mathrm{H}, 1}=-(1 / 2) \arcsin (1 / \gamma)$, the fixed point $\left(V^{*}, U^{*}\right)=$ $(0,0)$ loses stability via a Hopf bifurcation. Upon 


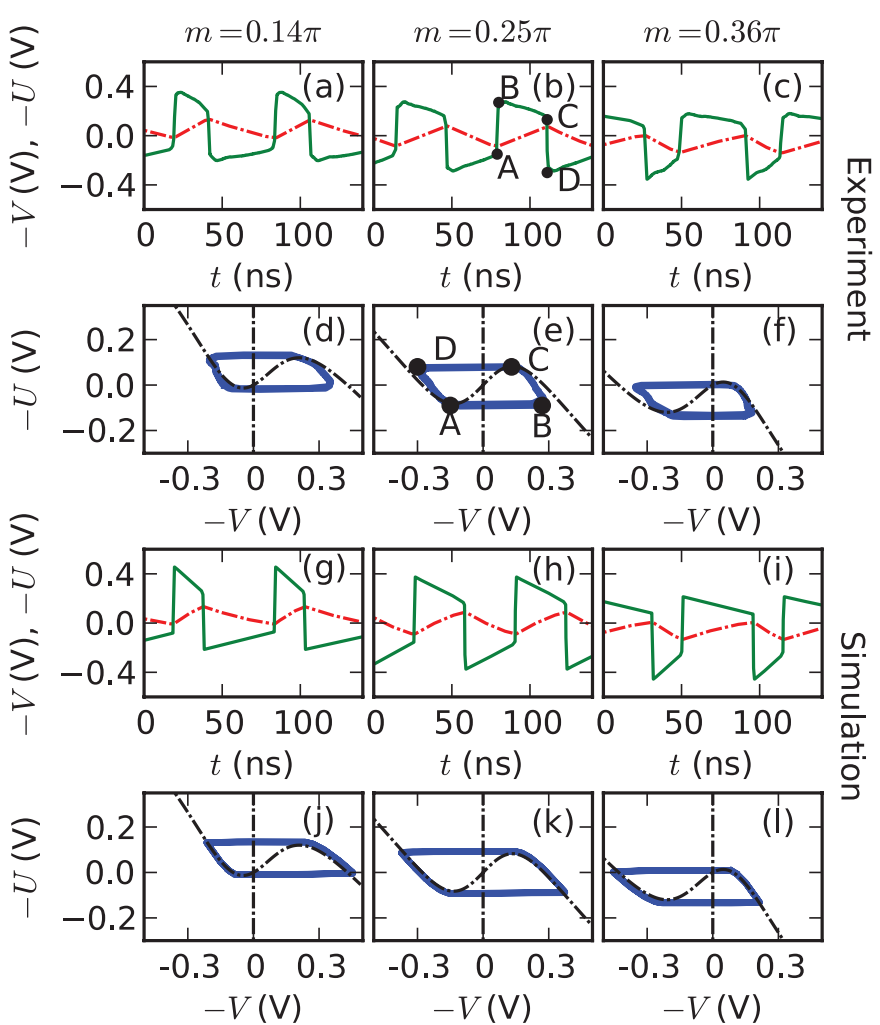

Fig. 4: (Color online) (a)-(c) Experimental waveforms $V(t)$ (green line) and $U(t)$ (red dashed line) for (a) $m=0.14 \pi$, (b) $m=0.25 \pi$, (c) $m=0.36 \pi$. (d)-(f) Respective phase space portraits (blue line: trajectory; black dashed lines: nullclines). (g)-(i) Numerical waveforms and (j)-(l) numerical phase portraits with same $m$ values. The other parameters are $\gamma=-2.08\left(m_{\mathrm{H}, 1} \approx 0.08 \pi\right.$ and $\left.m_{\mathrm{H}, 2} \approx 0.42 \pi\right), \tau=65 \mathrm{~ns}, d=1.0$, $g=-0.28 \mathrm{~V}$, and filter \#4 (see table 1 ). The experimental time series were averaged over 20 waveforms.

increasing $m$, a second Hopf bifurcation occurs at $m_{\mathrm{H}, 2}=\pi / 2+(1 / 2) \arcsin (1 / \gamma)$ (for $\left.\gamma<0\right)$, beyond which the system becomes again excitable. Both Hopf bifurcation points follow from linear stability analysis [16]. The Hopf bifurcations occur when the minimum or maximum of the nonlinear nullcline (eq. (4a)), respectively, shifts across the fixed point $(0,0)$, which can be used to analytically calculate $m_{\mathrm{H}, 1}$ and $m_{\mathrm{H}, 2}$. When the fixed point is located on the middle branch of the nonlinear nullcline, the system is in the oscillatory regime. As shown by Kouomou et al. [21], similar oscillatory states lead to breather solutions as $|\gamma|$ is increased past $\sim 3$.

To explore the oscillatory dynamics, we start in the pulsating state and tune $m$ past $m_{\mathrm{H}, 1}$. In figs. $4(\mathrm{a})-(\mathrm{c})$, $m$ is varied from $m=0.14 \pi$ to $m=0.25 \pi$ to $m=0.36 \pi$. At $m=0.25 \pi, V$ spends an equal amount of time at both the high and low levels. For $m=0.14 \pi$ and $m=0.36 \pi$, however, the duration of the high and low levels are no longer equal. The experimental time series (figs. 4(a)-(c)) and associated phase portraits (figs. 4(d)-(f)) agree with numerical time series (figs. $4(\mathrm{~g})-(\mathrm{i}))$ and phase portraits (figs. 4(j)-(l)).
From the phase portraits, it can be seen that the trajectory still follows the left and right branch of the nonlinear nullcline (eq. (4a)). With increasing $m$, the nonlinear nullcline shifts from right to left. When the fixed point is close to the minimum or maximum of the nonlinear nullcline, the trajectory slows down because the magnitude of the velocity field $(\dot{V}, \dot{U})$ is low in the vicinity of the fixed point. Thus, when the system is close to either Hopf bifurcation, the trajectory is expected to stay much longer on the left or right branch, respectively. Therefore, by changing $m$, we can control the duty cycle, which is defined as the ratio between time spent in an active state (i.e., the right branch of the nonlinear nullcline) and the period of the oscillation. For pulsing dynamics, the duty cycle $=w / \tau \propto 1 /(\tau \Delta \varepsilon) \approx 1 /\left(\tau \omega_{-}\right)$is scale invariant when increasing $\omega_{-}$and decreasing $\tau$ while keeping $\tau \omega_{-}=$const.

A quantitative analytical prediction of waveform and period in the oscillatory regime is possible in the vicinity of $m=0.25 \pi$, with a similar approximation as in eq. (7). The time-delayed feedback in eq. (2a) forces the trajectory to be $\tau$-periodic $(V(t-\tau / n)=V(t)$, with $n \in \mathbb{N})$. We find that a change in the low-frequency cutoff $\omega_{-}$changes the period of the oscillations through discrete changes in $n$.

Discussion and conclusion. - In an optoelectronic time-delay oscillator, we find novel pulse-train solutions with controllable pulse widths. The trajectories of these pulses are described with a nullcline analysis, where we assume $\tau$-periodic solutions in order to obtain simple equations for the nullclines. Because the trajectories in the pulsing and oscillatory states follow the nonlinear nullcline, analytic studies of the dynamics are possible.

Furthermore, we find that the periodic nullclines of our system are qualitatively similar to those of the FitzHugh-Nagumo model, which is a fundamental model for excitability in neurodynamics [22]. We also observe pulsating and oscillatory states similar to those found in simulations of the FHN model [11].

For the pulse-train solutions, we derive analytically the pulse width $w$ and show that it is inversely proportional to $\omega_{-}$. Earlier investigations have shown that the existence of a low-frequency cutoff is important when modeling accoupled systems [12-16], and we now add a quantitative dependence between the dynamics and $\omega_{-}$. This knowledge allows us to control $w$ of the pulses, which is verified by both experiments and simulations. We also produce pulses as narrow as $\sim 150 \mathrm{ps}$, and we conjecture that it is possible to tune the pulse widths even narrower with higher values of $\omega_{-}$. We show that the duty cycle is time scale invariant in the pulsing regime and can be tuned by changing the parameter $m$, and thus the nullclines, in the oscillatory regime. On a related note, Chembo et al. [23] already produced $4 \mathrm{ps}$ pulses in a more complex OEO that relies on a fine-tuned balance between dispersion and nonlinearity in the system.

Pulse trains with pulse widths on the order of $100 \mathrm{ps}$ correspond to microwave frequency combs, which can be 


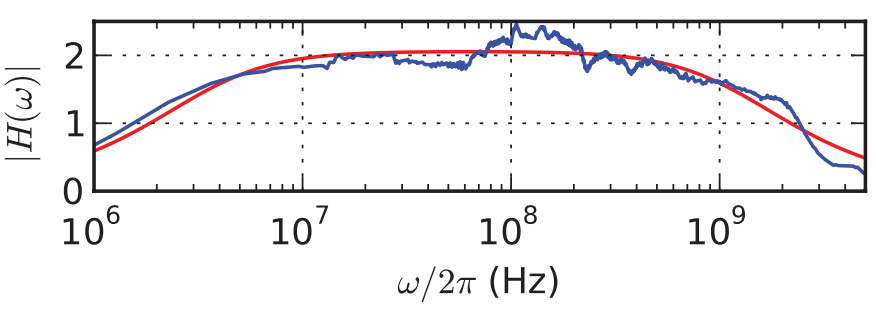

Fig. 5: (Color online) Blue dots: linear transfer function of our system using filter \#4. Red line: least square fit of a twopole bandpass filter with fit parameters $\omega_{-} /(2 \pi)=3.32 \mathrm{MHz}$, $\omega_{+} /(2 \pi)=1.22 \mathrm{GHz}$ and $\gamma=2.05$. The transfer function is measured with a linear spacing of 2 points $/ \mathrm{MHz}$ and fit with an exponential weight $\propto \exp (-\omega)$.

used in a variety of applications. The excitability and multistability characteristics of this system could also be exploited to build an optoelectronic memory [24], where the stored binary code travels as electronic and optical pulses around the delay loop. Coupling multiple OEOs in the excitable regime could lead to an artificial neural network. Small networks motifs of FHN models were already theoretically analyzed [10], but not experimentally realized to date. Networks of coupled OEOs in the excitable regime are also interesting from a computational point of view because coupled excitable systems could be used to form logic gates without a ruling clock [25].

$$
* * *
$$

DPR thanks Duke University for their hospitality, and acknowledges support by DAAD and TU Berlin. KEC and DJG gratefully acknowledge the financial support of the U.S. Office of Naval Research (N00014-07-1-0734). DPR and ES acknowledge support from DFG in the framework of SFB 910. We thank S. D. Cohen, H. L. D. DE S. Cavalcante and P. Hövel for fruitful discussions.

\section{ApPENDix}

We determine the feedback gain $\gamma$ by fitting the absolute value $|H(\omega)|$ of the transfer function of a two-pole bandpass filter described by

$$
H(\omega)=\gamma \frac{\Delta i \omega}{\omega_{0}^{2}-\omega^{2}+\Delta i \omega}
$$

to the frequency spectrum of our system in the linear regime $(m=-\pi / 4)$, as shown in fig. 5 . The maximum difference between the transfer function and the fit is $\approx 19 \%$ for $\omega /(2 \pi)=105 \mathrm{MHz}$ and is one source of error.

The parameters $d$ and $g$ are measured by stimulating the modulator driver and the MZM individually with a sine wave and recording the transmitted signal with an oscilloscope. Both parameters display weak frequency dependence, which is not captured by our model and indicates that the impedance of these devices is not precisely equal to $50 \Omega$. Using this method, the best experimental estimates are $d=1.0 \pm 0.05$ and $g=(-0.22 \pm 0.03) \mathrm{V}$. We expect that the values of these parameters will change somewhat when the components are incorporated into the system because of the variation in impedance of our dynamical system and the measurement apparatus. We find that the fit of our data to the nullclines is most sensitive to variation in $g$ and hence adjust its value to $g=-0.28 \mathrm{~V}$ to obtain the best agreement between experiment and observation.

\section{REFERENCES}

[1] Keener J. and Sneyd J., Mathematical Physiology (Springer, New York) 1998.

[2] FitzHugh R., Bull. Math. Biol., 17 (1955) 257; Nagumo J., Arimoto S. and Yoshizawa S., Proc. IRE, 50 (1962) 2061.

[3] Plaxa F., Velarde M. G., Arecchi F. T., Boccaletti S. and Ciofini M., Europhys. Lett., 38 (1997) 85.

[4] Gomila D., Matias M. and Colet P., Phys. Rev. Lett., 94 (2005) 063905.

[5] Bache M., Prati F., Tissoni G., Kheradmand R., Lugiato L. A., Protsenko I. and Brambilla M., Appl. Phys. B, 81 (2005) 913.

[6] Genevet P., Barland S., Giudici M. and Tredicce J., Phys. Rev. Lett., 101 (2008) 123905.

[7] Udem T., Holzwarth R. and Hänsch T., Nature, 416 (2002) 233.

[8] JuAn Y.-S. and Lin F.-Y., Opt. Express, 17 (2009) 18596.

[9] Dahlem M. A., Hiller G., Panchuk A. and Schöll E., Int. J. Bifurcat. Chaos, 19 (2009) 745.

[10] Schöll E., Hiller G., Hövel P. and Dahlem M. A., Philos. Trans. R. Soc. London, Ser. A, 367 (2009) 1079.

[11] Brandstetter S. A., Dahlem M. A. and Schöll E., Philos. Trans. R. Soc. London, Ser. A, 368 (2010) 391.

[12] Udaltsov V. S., Larger L., Goedgebuer J. P., Lee M. W., Genin E. and Rhodes W. T., IEEE Trans. Circuits Syst. I, 49 (2002) 1006.

[13] Goedgebuer J. P., Levy P., Larger L., Chen C. C. and Rhodes W. T., IEEE J. Quantum Electron., 38 (2002) 1178.

[14] Illing L. and Gauthier D. J., Physica D, 210 (2005) 180.

[15] Illing L. and Gauthier D. J., Chaos, 16 (2006) 033119.

[16] Callan K. E., Illing L., Gao Z., Gauthier D. J. and SchÖll E., Phys. Rev. Lett., 104 (2010) 113901.

[17] Peil M., Jacquot M., Chembo Y. K., Larger L. and Erneux T., Phys. Rev. E, 79 (2009) 026208.

[18] Illing L., Hoth G., Shareshian L. and May C., Phys. Rev. E, 83 (2011) 026107.

[19] Guglielmi N. and Hairen E., User guide for the code radar5-version 2.1 (2005) http://www. unige.ch/ hairer/software.html.

[20] Larger L. and Dudley J., Nature, 465 (2010) 41.

[21] Kouomou Y., Colet P., Larger L. and Gastaud N., Phys. Rev. Lett., 95 (2005) 203903.

[22] Izhikevich E. M., Dynamical Systems in Neuroscience: The Geometry of Excitability and Bursting (The MIT Press, Cambridge) 2007.

[23] Chembo Y. K., Hmima A., Lacourt P. A., Larger L. and Dudley J. M., Lightwave Technol., 27 (2009) 5160.

[24] Foss J., Longtin A., Mensour B. and Milton J., Phys. Rev. Lett., 76 (1996) 708.

[25] Motoike I. and Yoshikawa K., Phys. Rev. E, 59 (1999) 5354. 